\title{
Dining in Victoria, British Columbia: Vegetarianism and the Unaccommodating Restaurant Industry
}

\author{
Paul K. N. Shand
}

\begin{abstract}
This paper, a work-in-progress, uses the dining experiences of two vegetarians in Victoria, British Columbia to explore the unequal treatment they generally receive from Victoria's dining industry. Even though the participants felt that Victoria is a great place for vegetarians, findings from three interviews, four food menus, and my direct experience as a cook suggest that the restaurant industry - which is primarily geared towards nonvegetarians - treats diners in Victoria unequally, especially vegetarians. By identifying vegetarians as a distinct social group this project shows how inequality against vegetarians in Victoria operates. The thesis of the research project, however, cannot be generalized to include the larger body of vegetarians in Victoria because of the small sample group. This research project recognizes that far more interviews and further research are needed to verify its hypothesis.
\end{abstract}

Key Terms: vegetarian, social space, inequality, restaurant industry, food hierarchy, dominant food culture

\section{Introduction}

As a professional cook in Victoria, I have observed many restaurants offer minimal accommodations for vegetarians. Consequently I set out to investigate the unequal treatment of vegetarians by examining individual experiences of dining out in Victoria. In this "in-progress" research project, I rely on the perspectives of two vegetarians, identified as Candace and Dawn to protect their privacy. I contextualize their perspectives by also incorporating the viewpoint of a cook (Oscar), analyzing a number of menus, and, when necessary, referencing my own culinary experience. Contrary to what the two vegetarians believe, the collected data show that the restaurant industry, primarily geared towards a non-vegetarian population, does not equally accommodate vegetarians. Consequently, I hypothesize that the two vegetarian participants - and maybe the larger body of vegetarians in Victoria - are forced into a distinct, marginalized, social group. But 
first, from what angles does the available literature approach food and vegetarian diners?

Little attention has been paid to studying inequality against individuals or groups who choose a specific diet. Most literature focuses on food consumption as an expression of cultural membership (Caplan, 1994; Goody, 1982; Inness, 2006; Mennel, Murcott, \& van Otterloo, 1992), investigates minorities' access to food in specific sectors of the restaurant industry (Freeman, 2007), explains eating in relation to the physical space in which it occurs (Bell \& Valentine, 1997), and researches the social activity of eating in restaurants (Finkelstein, 1989). As Finkelstein (1989) established, dining out is an important social event. This study expands on Finkelstein's work, specifically looking at how vegetarians experience inequality when they dine out. By focusing on vegetarians, I examine food from a different angle: rather than treating dining habits as a representation of existing cultural distinctions between social groups, with vegetarianism at the center, this study views diet as the cause of inequality between vegetarians and nonvegetarians.

\section{Discussion}

Are vegetarians a distinct social group? The fact that "vegetarian" has become a common descriptor establishes them as a social group. Bourdieu (1986) explains that a group may have a distinct identity that can "be socially instituted and guaranteed by the application of a common name" (p. 249). This leads to the creation of a specific social space for vegetarians. Importantly, the distinction between "vegetarian" and "non-vegetarian" is connected with levels of social inclusion: vegetarians are less included and separate from the socially normal, dominant non-vegetarian group. The inclusion/exclusion dichotomy is what makes inequality possible. The non-vegetarian group rewards its members for conforming but rewards are unavailable to the excluded group, in this case, the vegetarians. In Victoria's restaurant industry the rewards manifest as levels of accommodation. Diners who are vegetarian are offered fewer food options and are excluded from the dominant group. Initially both vegetarian participants, Candace and Dawn, said that Victoria is a great place for vegetarians. However, I found evidence that there is a distinction between vegetarians and non-vegetarians, and that the nature of the relationship between the two groups is unequal.

Candace and Dawn both mentioned that Victoria is an "easy" place to be vegetarian. Candace said "it's easy to be vegetarian in Victoria... there's a lot more options than there used to be" and Dawn said, "in Victoria it's super easy, every restaurant has at least a couple vegetarian options here." They were both happy 
with their choice and felt that most establishments offered them something to eat. However, when asked to give specific examples of the food that is usually available both said they often just order the salad or a veggie burger. In addition, both vegetarian participants have started to eat fish regularly, in foods such as sushi, because of nutrition and convenience. On one hand, this suggests that it is difficult to maintain a healthy vegetarian diet, even in Victoria where they had said it is quite easy. But, this discrepancy might be better explained by the inequality of treatment offered by Victoria's restaurant industry to vegetarians as compared with non-vegetarians. It is the distinction between these two groups that allows for the inequality in the first place.

Indeed, two main themes in my data indicate that vegetarians are a distinct, yet marginalized, social group in Victoria: a) Victoria has an established vegetarian social group, and b) a person requires certain personal traits to enjoy vegetarianism. Vegetarian venues support social networks that allow vegetarians to develop a sense of belonging to a community, a safe and comfortable place where one need not be forceful to get decent food. In these places vegetarians feel "normal" because most diners have similar diets. Dawn said, "I know there is a vegetarian culture in Victoria." Both vegetarians' interviews suggested the existence of a common identity and distinct social space for vegetarians. Candace noted that "we have a ton of vegetarians in my family," while Dawn explained that people who are vegetarian are also likely to agree on other matters: "it's not a coincidence that there are a lot of vegetarians in my social circle," she said. These findings parallel Bourdieu's (1986) theories that people who have similar cultural tendencies will likely occupy a common social space. Candace and Dawn, however, mentioned they had been excluded from a group because of being vegetarian. Candace said, "a lot of the time people go out and they want to share 'appys,' I can't really do that" and Dawn said "sometimes it could be a bit awkward (being vegetarian)... but it doesn't bother me, I'm used to it by now." Because of the insufficient accommodation offered by many restaurants, both vegetarians felt different from others. Furthering the idea of a social separation, Candace stated that "there are veggie restaurants in town," indicating a difference between establishments who welcome vegetarians and those who do not.

I assert that the unequal treatment in Victoria's restaurant industry may have led Candace and Dawn to believe that becoming a successful vegetarian locally requires developing a particular type of personality. Candace said, "it totally depends on your personality how successful it's going to be... I'm an extremely blunt person." Dawn said, sometimes "there's negotiations with the kitchen." Choosing vegetarianism requires confidence and not being embarrassed by the 
limitations encountered in accessing the appropriate foods. In my experience, servers and cooks will often associate a sense of nuisance or even disdain people who wish to alter the menu choices or ingredients. Oscar suggests that he sees vegetarians as different from non-vegetarians, stating that they often require more work, creativity, and sometimes disrupt the production of food at meal times. If customers demand vegetarian food, they are more likely to get it because it will not always be offered initially. However, the inequality in the treatment of Candace and Dawn in Victoria cannot only be explained by the logistics of running a restaurant.

Though it would be feasible to fully accommodate vegetarians, many restaurants do not. As a professional cook for many years, I know that planning a menu that will better accommodate vegetarians is simple and cost effective, because vegetables are cheaper than meat. Still, many restaurants continue to limit the level of service they offer to vegetarians. I selected four of the forty-dollar menus available during Dine Around Victoria 2012 to represent the range of establishments that participated in the event. The Dine Around 2012 menus, which have only three options for each course, showcase the availability level of vegetarian food in Victoria's restaurant industry. These menus are carefully designed to appeal to large numbers of people, and aim to portray local restaurants as more accommodating to vegetarians than they are usually. I have observed that whether the menu has three or ten choices there will often be only one or two vegetarian options, much less than meat and fish. Some of the menus have no vegetarian options other than dessert, and only half of the menus have a vegetarian main course option.

The lack of choice on Dine Around 2012 menus is not the only way that inequality against vegetarians takes place. All participants spoke of experiencing anti-vegetarian sentiment in certain restaurants. Oscar suggests that vegetarians' failure to conform to the norm results in inferior treatment from the restaurant industry. He said, "if vegetarians want good food they should eat at a vegetarian place." But Oscar also said he enjoys the challenge of cooking for vegetarians: "it does bring out like the creative aspect of some cooks." The discrepancy in his statements might explain how individual cooks can be sympathetic towards the needs of vegetarians while the industry continues to exclude them. As Oscar said, "it's kinda weird that vegetarians get the flack."

But further than specific experiences in restaurants, the inequality between vegetarianism and non-vegetarianism in Victoria involves a hierarchal relationship. This may privilege people who eat meat over vegetarians. And, as Oscar indicated, at a vegetarian restaurant it is likely "that the guys in the back (the cooks) aren't omnivores." As well, my own experience has shown that a cook who is vegetarian 
is not taken as seriously or seen as having as high a level of skill as one who eats meat. Also, when listing the names of high-end restaurants that Candace attends for professional reasons, she mentioned no vegetarian restaurants. Likewise, Dawn did not mention vegetarian restaurants as destinations for a celebration dinner, while Oscar ranks Victoria's vegetarian restaurants as only moderately prestigious. Evidence suggests a link between eating meat and social status in the form of "prestige." In a list of high-end ingredients given by Oscar, all are meat or fish except for truffles. To Candace, "meat is much more expensive than veggie food." Dawn likewise noted that meat is more expensive than vegetarian food. Both statements agree with Bourdieu's (1986) concept of economic capital: higher quality and more prestigious food costs more money.

\section{Conclusion}

Both Candace and Dawn have to justify to friends and servers why they are vegetarian. Dawn said, "you often have to justify why you eat (vegetarian).... I shouldn't have to justify why I choose to eat the way I do." The need to justify one's diet indicates that vegetarians in Victoria may occupy a lower spot on the social hierarchy than non-vegetarians. The underlying logic being that those who are in a powerful position do not have to justify it, while people who choose to be different need to explain. Because vegetarianism is viewed as a dietary choice, those who choose it have to explain why. It is not as easy, functionally and socially, as Dawn and Candace think to maintain a vegetarian lifestyle in Victoria. Hence this study concludes that vegetarianism may be the basis for inequality in Victoria's restaurant industry. However, it is important to note that this is not a large study and its findings cannot be generalized. A question that requires further investigation is: who determines what food culture is? Regardless, more research must be done to comprehensively explore and establish these findings beyond the limited scope of this project.

\section{References}

Bell, D. and G. Valentine. (1997). Consuming geographies: We are where we eat. New York, NY: Routledge.

Bourdieu, P. (1986). The forms of capital. In J. G. Richardson (ed.), Handbook of theory and research for the sociology of education (pp. 241-258). New York, NY: Greenwood Press.

Bourdieu, P. (1989). Social space and symbolic power. Sociological Theory, 7(1), $14-25$. 
Caplan, P. (1994). Feasts, fasts, famine: Food for thought. Oxford, England: Berg. Finkelstein, J. (1989). Dining out: A sociology of modern manners. Cambrige, England: Polity Press.

Freeman, A. (2007). Fast food: Oppression through poor nutrition. California Law Review. 95(6), pp. 2221-2259.

Goody, J. (1982). Cooking, Cuisine and Class: A study in comparative sociology. Cambridge, England: Cambridge University Press.

Inness, S. A. (2006). Secret ingredients. New York, NY: Palgrave MacMillan.

Mennel, S., Murcott, A., \& van Otterloo, A.H. (1992). The sociology of food: eating, diet and culture. Thousand Oaks, CA: Sage.

Tourism Victoria. (n.d.). Retrieved March 7, 2012, from Tourism Victoria website, http://www.tourismvictoria.com/dine

\section{Contact Information}

Paul K. N. Shand, from the Department of Sociology, can be reached at paulshand@ymail.com.

\section{Acknowledgements}

I would like to express my thanks and gratitude to those who made this research project possible. I am grateful to Dr. André Smith in the Department of Sociology at the University of Victoria. Dr. Smith spent many hours consulting and encouraging me and without his support this project would not have been possible. I would also like to thank the people in Victoria's restaurant industry who in one way or another contributed to this research. Finally I would like to thank the participants of this study who graciously shared with me their personal experiences. 